Part 1. Jets basic issues and physical proccesses 


\title{
The formation of relativistic cosmic jets
}

\author{
David L. Meier ${ }^{1}$ \\ ${ }^{1}$ MS 169-506, Jet Propulsion Laboratory, California Institute of Technology, \\ 4800 Oak Grove Drive, Pasadena, CA 91109, USA \\ email: David.L.Meier@jpl.nasa.gov
}

\begin{abstract}
I review current ideas on the launching, acceleration, collimation and propagation of relativistic jets and the influence of strong magnetic fields in the process. Recently, several important elements of the entire jet "engine" structure have been shown to play key roles in the production of an astrophysical jet. Depending on the type of system, these include the spin of the central black hole, the thermal and/or magnetic state of the accretion flow, the presence of a re-collimation point in the jet outflow far away from the central object, and the behavior of MHD shocks and kink instabilities in the final jet. While these physical processes probably are at work in all types of relativistic jets (and many even in more benign stellar outflows), I shall concentrate on ones produced by lower luminosity black hole sources, both in active galactic nuclei and in X-ray binaries. I also will discuss the connection between the theoretical concepts and the large body of observational data now available on these systems.
\end{abstract}

Keywords. black hole physics, accretion, MHD, galaxies: jets, X-ray binaries: jets

\section{Introduction}

Despite the tremendous theoretical and numerical progress made in the past $>40$ years since the first models of pulsar "winds" as outflows driven by rotating magnetic fields, many astronomers believe that we still have no idea how cosmic jets are produced. Actually, we really do know a lot about the subject, and we have very good ideas about most of the missing pieces of the puzzle. The purpose of this paper is to outline what we do know, what we strongly suspect, and what aspects of jet production remain a mystery.

While this work will concentrate on the production of relativistic jets, much of it (especially Sections 3, 4, and 5) are relevant to jets on all scales. But, before beginning the discussion, it is very important to define for the reader what we mean by a "cosmic jet" (even though this definition is clear to most attendees of this conference). A cosmic jet is an extraordinarily large scale, coordinated, virtually universally bipolar and highly supersonic outflow of plasma from the region near a central gravitating star. Jets frequently, but not universally, involve accretion of outside material onto that star. The acceleration and collimation zone (ACZ) alone is typically 100 - 1000 time the "injection" radius where most of the outflow originates, and the latter can be larger than the star itself. For example, while the sun no longer produces such a large-scale jet, it almost certainly did so when it was a protostar 4.6 Gyr ago. The ACZ extended perhaps $1-10 \mathrm{AU}$ above the protoplanetary disk ecliptic plane, and the length of the full jet was a fair fraction of a parsec. Such jets dwarf, by $6-7$ orders of magnitude in size and power, phenomena such as solar flare jets and even coronal mass ejections.

Below we cover five issues in the launching, acceleration, and collimation of jets. We begin by discussing the role of the central rotating object itself and end with the behavior of the freely-propagating jet flow, far from the central object and causally disconnected from the jet engine itself. 


\section{The Role of the Central Rotating Star}

Our discussion of the role of the central rotating, gravitating object in launching the jet will be oriented specifically toward the relativistic jet case. In fact, we shall deal only with black holes and not with neutron stars in this review. The main question here is, how important is the rotating black hole in driving the jet (i.e., the Blandford-Znajek process [Blandford \& Znajek 1977, hereinafter BZ]) compared to the rotating accretion disk (i.e., the Blandford-Payne process [Blandford \& Payne 1982, hereinafter BP]).

Black holes alone cannot support magnetic fields. While a spinning and charged (i.e., Kerr-Newman) black hole can have a magnetic moment, the amount of charge expected on astrophysical black holes is extremely small (BZ). Therefore, any black hole magnetosphere must be anchored in the accretion inflow itself, where strong currents can be maintained (BZ; Punsly \& Coroniti 1990).

Closed vs. open magnetospheres. In a steady state we expect two different types of magnetosphere: closed (see, e.g., Uzdensky 2005), with no open field lines penetrating the black hole horizon, and open (see, e.g., Garofalo 2009), with significant amounts of magnetic flux deposited on the horizon. While both types of magnetosphere can extract angular momentum from the black hole, the ability of each in driving a jet is quite different. The closed magnetosphere deposits black hole angular momentum into the accretion disk, where it can be transferred radially outward through via viscous processes. The open magnetosphere, on the other hand, extracts angular momentum to vertical infinity in a large-scale torsional Alfvén wave (a Poynting flux jet). To first order, then, open black hole magnetospheres should produce jets, while closed ones should not.

Strong vs. weak shear and the magnetic tower effect. Whether or not a closed magnetosphere will remain so (and not produce a jet) will, in turn, depend on the amount of shear between the black hole and the accretion disk - specifically between the horizon and the point on the accretion disk where the other footpoint of the closed field loop is anchored. If the shear is high, the magnetic tower process (Lynden-Bell 1996; Meier et al. 1997; Romanova et al. 1998; Uzdensky \& MacFadyen 2006) will cause the closed field loops to inflate vertically and break, converting a closed magnetosphere into an open, jet-producing one. Since most of the field loop outer footprints lie near the disk innermost stable circular orbit (ISCO), we will approximate the shear as simply the absolute value of the angular velocity difference between the horizon and the ISCO, and normalize the value to that of a non-rotating Schwarzschild black hole

$$
\Sigma=6^{3 / 2}\left|\left(j / 2 r_{\mathrm{h}}\right)-1 /\left(r_{\text {isco }}^{3 / 2}+j\right)\right|
$$

where $r_{\mathrm{h}}$ and $r_{\text {isco }}$ are the standard horizon and ISCO radii in units of the gravitational radius $r_{g}=G M / c^{2}$ and are functions of the normalized black hole spin $j=J c / G M^{2}$, which ranges between -1 and +1 .

The strongest shear occurs for black holes spinning in the opposite sense to the accretion disk $(-1<j<0)$ and for a small range of fairly rapid prograde holes $(0.75<j<0.99)$. The weakest shear occurs for $0<j<0.75$ and for $j>0.99$ (Meier \& Garofalo 2010), with zero shear occurring at $j \approx 0.36$ and $j=1.0$. Therefore, black-hole-driven BZ jets are most likely to form in retrograde black hole systems, while prograde holes (with an order of magnitude or less shear) are least likely to produce BZ jets.

Jet power vs. black hole spin. In addition to determining the likelihood of producing a jet, black hole spin also determines the power of that jet, and this process operates in the same sense: retrograde black holes produce the strongest jets by $1.5-2$ orders of magnitude (Garofalo 2009; Garofalo et al. 2010). This effect is due to magnetic flux in the plunging region $\left(r<r_{\text {isco }}\right)$ rapidly accreting onto the black hole. That is, flux that 
would have been located in the "gap" region between the horizon and ISCO instead ends up on the horizon. Retrograde black holes have large gaps, up to $8 G M / c^{2} v s$. as low as $\sim 0 G M / c^{2}$ for maximum prograde Kerr holes. Since the jet power is proportional to the square of the horizon flux (BZ), this leads to much stronger BZ jets from retrograde systems than prograde ones.

The combined effect of shear and horizon magnetic flux creates a gross spin asymmetry in the production of jets by spinning black holes: retrograde holes should produce strong, radio loud sources, while prograde holes should produce radio quiet sources, or even radio silent ones in the nearly zero shear cases.

A modified spin paradigm. These results suggest a "spin paradigm" that differs significantly from that suggested previously (Wilson \& Colbert 1995; Meier 1999). While rapid black hole spin is necessary for producing a radio loud source, it is not sufficient. That rapid spin also must be retrograde. Rapid prograde spin produces radio quiet sources. In terms of the Sikora et al. (2007) picture of radio loud and quiet sources, one would conclude that Fanaroff \& Riley class I sources (FR Is), broad line radio radio galaxies (BLRGs), and radio-loud quasars (FR IIs \& RLQs) have primarily retrograde black holes. On the other hand, low-ionization nuclear emission-line regions (LINERs), Seyfert galaxies, and Palomar-Green (PG) quasars have primarily prograde black holes.

Solution to the spin paradox. Under the old spin paradigm (rapid spin produces powerful radio jets) a paradox arose. Because few powerful FR II radio sources exist at the present epoch, the old paradigm implied that black holes now spin slowly. However, optical statistical studies have concluded that black holes now spin rapidly (Yu \& Tremaine 2002; Elvis et al. 2002). Furthermore, the few sources that have measured rapid prograde spin are radio silent (Iwasawa et al. 1996; Brenneman \& Reynolds 2006; Fabian et al. 2009), while powerful radio sources with measured ISCOs show a very large gap/plunging region (Kataoka et al. 2007; Sambruna et al. 2009). This all can be explained by the new paradigm: black holes do indeed rotate rapidly now, but in a prograde sense, rendering most radio quiet. And it is quite possible that radio loud objects reveal a large gap because they have retrograde spin.

In short, therefore, black hole systems (at least supermassive ones) show strong evidence of black-hole-driven BZ jets when the spin state is of the correct sign (retrograde).

\section{The Role of the Rotating Accretion Disk}

Because jet outflows also can be driven by rotating, magnetized accretion disks (BP; Li et al. 1992; Vlahakis et al. 2000; Vlahakis \& Konigl 2003), it is appropriate to ask if many of the jets emanating from black hole systems are disk, rather than black hole, driven. Indeed, after an extensive study of microquasars in X-ray binary systems, Fender et al. (2010) concluded that in the X-ray hard state there is no evidence for any correlation between the jets produced and the reported spin measurements of the black hole in those binary systems. While this is indeed an interesting result, it is far too early to conclude that black hole spin plays no role in X-ray binary systems for the following reasons:

- Absence of evidence is not necessarily evidence of absence.

- Black hole spin measurements of the same source vary greatly; e.g., GRS 1915+105 measurements range from 0 to 1 .

- Just because a jet property clearly changes with the state of the accretion flow does not mean that the jet is driven by the rotating disk. Recall (see above) that the BZ process depends on the state of the magnetosphere, which is anchored in, and created by, the accretion flow. 
- A black-hole-spin-driven jet may be very relativistic $(\gamma>10)$ and, therefore, highly beamed. Unless an observer views such a jet within a few degrees of its axis, it may be largely invisible to observers but nevertheless important in the overall source energetics.

Nevertheless, it is important to take stock of accretion disk jet launching theory and attempt to compare with observations, particularly those in hard accretion states.

Jets from advection dominated accretion flows (ADAFs). The ADAF is the leading model for hard state accretion flows, so it is important to assess its ability to produce the jets observed in hard state objects. McClintock \& Remillard (2006) published both photon and power density spectra (PDS) of several microquasars in the hard state (their Figs. $4.11 \& 4.12$ ). While there are several accretion models for producing a hard powerlaw photon spectrum up to $100 \mathrm{keV}$, the PDS affords a glimpse into the turbulent state of the accretion flow that cannot be seen in the photon spectrum alone. If we assume that the observed power spectrum is composed of many individual power spectra at different disk radii $R$, then the power contributed at a frequency $f=V_{\text {turb }} / H$ (where the velocity of the largest eddy is proportional to the sound speed $V_{t u r b} \propto c_{s} \propto R^{-1 / 2}$ and and the disk half-thickness $H \propto R$ in an ADAF model) is given by $d \mathcal{P}=2 \pi R 2 H d R \rho V_{\text {turb }}^{2}$. The total power per unit frequency then will vary as

$$
d \mathcal{P} / d f \propto f^{-4 / 3}
$$

Most of the McClintock \& Remillard power density spectra have slopes between -1.1 and -1.4 and extend up to several hundred $\mathrm{Hz}$ - very similar to what is expected from an ADAF whose turbulence extends down to the ISCO of a $10 M_{\odot}$ black hole.

A very nice demonstration of how ADAF-like accretion flows can drive jets was performed in a tour de force simulation by McKinney (2006). Like many prior magnetorotational instability (MRI) simulations (e.g., McKinney \& Gammie 2004; Hawley \& Krolik 2006), McKinney's generated a collimated outflow from a hot, geometrically thick, radiatively inefficient, magnetized accretion flow. However, in this case he allowed the simulation to continue for $\sim 10^{4}$ gravitational times $\left(\tau_{g}=G M / c^{3}\right)$ and the jet to propagate to about $10^{4} r_{g}$. Both observationally and theoretically, therefore, ADAFs or similar flows appear to be a good explanation for steady jets observed in the hard state especially for those sources that show PDS up to hundreds of $\mathrm{Hz}$.

Jets from magnetically dominated accretion flows (MDAFs). For some of McClintock $\&$ Remillard's sources, most notably GRO J1655-40 in its hard state and GRS 1915+105 in its plateau state, the PDS has a sharp cutoff in turbulent noise above a few $\mathrm{Hz}$ plus a strong low-frequency quasi-periodic oscillation (LF QPO) just below the noise cutoff. $\dagger$ It has been suggested (Meier 2005; Fragile \& Meier 2009) that such a PDS can be best explained by the formation of a strongly-magnetized region inside a classical ADAF, with a size $r_{\text {mdaf }} \sim 10 r_{\text {isco }}$. A semi-rigid, rotating inner magnetosphere would account for both the noise bandwidth cutoff and the strong QPO at only a few Hz.

Jet mini-suppression in the hard state. MDAFs also predict a moderate suppression of the jet power of order $r_{\mathrm{mdaf}} / r_{\text {isco }} \approx 10$, since the ADAF jet in this case would be launched from a shallower region of the black hole potential well (10 vs. $\left.1 r_{\text {isco }}\right)$. When we factor in the observed jet radio power as a function of jet total power $\left(L_{R} \propto L_{J}^{1.3-1.4}\right.$, Migliari \& Fender 2006), this predicts radio power suppression between ADAFs and MDAFs of order $\sim 20-25$.

$\dagger$ Interestingly, GRS 1915+105 and XTE J1550-564 show similar bandwidth-limited noise and LF QPO in the very high/soft power-law state [McClintock \& Remillard 2006, Fig. 4.15], which may be the super-Eddington, radiation-pressure-dominated equivalent of the ADAF state. 
Fender et al. (2010) have discovered a new class of microquasars, which they call "outliers", that lie below the classical fundamental plane, with radio powers suppressed by a factor of $\sim 20$ compared with other hard-state microquasars with the same X-ray luminosity. $\ddagger$ (See also Gallo's and Soleri \& Fender's articles in this volume.) Curiously, and perhaps not coincidentally, GRO J1655-40 and XTE 1550-564 are outliers, and GRS $1915+105$ lies at the intersection of the outlier trend line and the fundamental plane. It is suggested here, therefore, that outliers are related to sources with bandwidth-limited PDS noise and may be related to accretion flows with MDAFs in the centers of their ADAFs. More work is needed to see if most sources in an "outlier" state also have bandwidthlimited noise when in that state. Why some sources show these features and why some do not is a complete mystery at present.

Jet suppression in the soft state. Suppression of the jet radio power by a factor of 50 or more occurs when sources transition from a hard state to a soft one (Fender 2001). This author (Meier 2001) suggested that this suppression occurs because the magnetic field vertical component (needed to drive a BP jet from the disk) is significantly smaller in cool, geometrically thin accretion disks than in hot, geometrically thick ADAFs. From standard disk/ADAF models, one predicts a suppression factor of $\sim 100-200$, consistent with observations. This suggests that all strong accretion disk driven (BP) black hole jets are produced only when the source is in a geometrically thick accretion state.

At first, this conjecture seems to be in contradiction with the generation of strong, "ballistic" and explosive jets when sources transition from the hard state to the soft state (Fender et al. 2004). The two can be reconciled, however, if we recall that at high accretion rates disks may be thermally and secularly unstable (Shakura \& Sunyaev 1976; Pringle 1976) and may exhibit limit-cycle behavior in which the disk temporarily becomes hot and geometrically thick, drains quickly, and then refills on an accretion time scale (Szuszkiewicz \& Miller 2001). In this case, a jet still would be generated in the geometrically thick hot state as it drains (the explosive jet) followed by a long period of recovery in a cooler, geometrically thin state. For GRS 1915+105 the predicted cycle time is $\sim 1000$ seconds, similar to the observed cycle time for the explosive jets when this source transitions from a hard to a soft state.

\section{Jet Acceleration and Collimation}

Once launched from the rotating compact object or from the rotating accretion flow, the outgoing magnetized plasma will be accelerated and collimated by the strong, twisting global magnetic field. Compared to the launching processes, the physics of jet acceleration and collimation is much better understood, due in no small part to the pioneering work of Blandford \& Payne (1982). They outlined the basic principles of MHD jet production assuming a steady state, axisymmetric, and spherically self-similar flow to turn a 3dimensional, time-dependent problem into a 1-dimensional one.

Many things have been said about the self-similar assumption made by BP, both favorable and unfavorable. This assumption is not the last word in jet theory by any means. More realistic and more complicated (but related) models and simulations (such as McKinney 2006) will follow in the coming years. Self-similarity also is not the physically implausible assumption that some have capriciously claimed. It simply is a convenient way to express external confinement of the greater jet engine by a poorly understood external medium (e.g., by the interstellar medium). With surprisingly little external

$\ddagger$ These also sometimes are called "radio quiet black holes", which is an inappropriate name, as it evokes memory of the radio quiet quasars, whose radio powers are $4-6$ orders of magnitude weaker than radio loud ones! 
gas pressure confinement, a large-scale rotating magnetosphere can attain a pseudo-selfsimilar structure interior to that external medium and external to the rotational axis.

An important parameter in these models is the run of current (and therefore magnetic field) with spherical radius $r$, which can be specified along the equator (i.e., near the accretion flow) in cylindrical coordinates

$$
F \equiv \partial \ln B / \partial R+2
$$

When $F>1$ (often called the forward current regime), the current carried by the jet increases with disk radius $R$; when $F<1$ (the return current regime), the current decreases with $R$; and when $F=1$, there is no current carried by the jet normal to the disk, only that carried in the radial $r$ direction. For all types of rotating, turbulent accretion flows (ADAF, slim [super-Eddington, radiation-pressure-dominated] disk, Shakura and Sunyaev outer and middle regions) $F \approx 0.75-0.81$ (the return current regime). So, for most accretion flow jets, return current is carried primarily by the portion of the jet that emanates from near the disk inner edge (shortly outside the ISCO), but inside that portion there must be a non-self-similar region that transitions to a forward current solution to keep the current on the rotation axis finite.

MHD wind/jet models are very similar in mathematical structure to the Parker hydrodynamic (HD) wind, just more complex because the equations are solved in the spherical $\theta$ direction, rather than in $r$, and because there are three characteristic waves and phase speeds instead of one. When the magnetic field is much stronger than the plasma dynamical forces (i.e., plasma $\beta_{\mathrm{p}}=p_{\text {gas }} / p_{\text {mag }}<1$ ), the three modes are

- Transverse Alfvén waves, which propagate along field lines with $V_{\text {phase }}=V_{A} \cos \chi$, $V_{A}=(B / 4 \pi \rho)^{1 / 2}$, and $\chi$ being the angle between the magnetic field and the propagation direction and $\rho$ being the plasma mass density.

- Longitudinal, fast magnetosonic (pressure) waves, which propagate fastest normal to the field at $V_{F} \approx\left(V_{A}^{2}+c_{s}^{2}\right)^{1 / 2}$.

- Longitudinal, slow magnetosonic waves, which propagate at $V_{\text {phase }}= \pm\left(c_{s} V_{A}\right)^{1 / 2}$ $\cos \chi$ - best along the field $(\chi=0)$ and not at all normal to it.

In a very nice paper Bogovalov (1994) outlined the physics of MHD winds in the general (non-self-similar) case. There are a total of six important surfaces in the flow:

- Three "critical surfaces" (the fast magnetosonic, slow magnetosonic, and cusp surfaces), across which information propagation in a given wave mode changes from being omni-directional to occurring along specific directions or caustics. We will not discuss these surfaces much in this paper.

- Three "separatrix surfaces", across which information propagation in a particular wave mode further changes from having components upstream and downstream in the plasma flow to having only one of these components. These are the Alfvén surface (AS), the fast magnetosonic separatrix surface (FMSS), and the slow magnetosonic separatrix surface (SMSS). Of these three, the FMSS is the most important and plays the same role as the sonic surface in the Parker wind. The FMSS is the magnetosonic horizon, beyond which no information can propagate back to the jet engine that accelerates the flow. $\dagger$

In a time-independent mathematical model, the separatrix surfaces become singular surfaces, where both numerator and denominator of the wind acceleration equation vanish simultaneously. And, if that theory also is 1-dimensional, the singular surfaces become simple singular points along a representative streamline. In the strong-field, MHD case, these points have the names "modified slow point" (MSP), "Alfvén point" (AP), and

$\dagger$ In the Parker HD wind model there is only one critical and one separatrix surface, and these coincide to form a single sonic surface where $V=c_{s}$. 
"modified fast point" (MFP). Setting the wind equation denominator to zero at these points generally determines the location of each surface; setting the numerator to zero determines the flow internal boundary (or regularity) conditions on those surfaces; and applying l'Hospital's rule to the ratio of numerator and denominator determines how the flow conditions cross these surfaces. Any viable steady wind/jet solution of the MHD flow must pass through all three separatrix/singular surfaces in a smooth manner.

Implications for jet acceleration and collimation. In an accelerating jet flow the physics is different in at different stages of the acceleration. From the launch point near the accretion disk or black hole up to the SMSS the flow will be accelerated primarily by gas pressure forces. Beyond that surface the plasma flow is too fast for hydrodynamic forces to act, but not for the rotating magnetic field (torsional Alfvén wave) to continue the acceleration up to the AS. By this time the magnetic field has developed a strong toroidal component, and magnetic pressure $\left(-d\left(B_{\phi}^{2} / 8 \pi\right) / d Z\right)$ assists in the acceleration. But soon the flow exceeds even the fast magnetosonic speed.

However, observed jet speeds far exceed their magnetosonic speeds. What produces the remaining acceleration? The self-similar theory reveals the answer: pinch forces $\left(-B_{\phi}^{2} \cot \theta / 4 \pi r\right)$ accelerate the flow forward and toward the axis until the $\theta$ component of the velocity exceeds the fast magnetosonic speed (i.e., $-V_{\theta}>V_{F}$ ) when it crosses the FMSS. There are several important points about this process:

- If the FMSS lies near the axis $\left(\theta_{\mathrm{FMSS}}<<1\right)$, then the jet speed $V_{Z} \approx V_{r}>>\left|V_{\theta}\right| \sim$ $V_{F}$; i.e., the jet speed far exceeds the fast magnetosound speed.

- In fact, if the parameter $\sigma \approx\left(V_{A}^{2} / V_{0} c\right)\left(R_{0}^{2} / R_{L}^{2}\right)>>1$ (where $V_{0}$ and $R_{0}$ are the velocity and radius at the base of a jet streamline, and $R_{L}$ is the light cylinder radius), very relativistic jet speeds can be attained (Michel 1969; Li et al. 1992; Polko et al. 2010).

- Since the flow toward the axis is supermagnetosonic, it will bounce or shock on the axis in less than a magnetosound crossing time.

- However, because the FMSS is the magnetosonic horizon, any shock or other feature in the jet flow there will not affect the ACZ. Beyond the FMSS the jet is causally disconnected from the engine that created it.

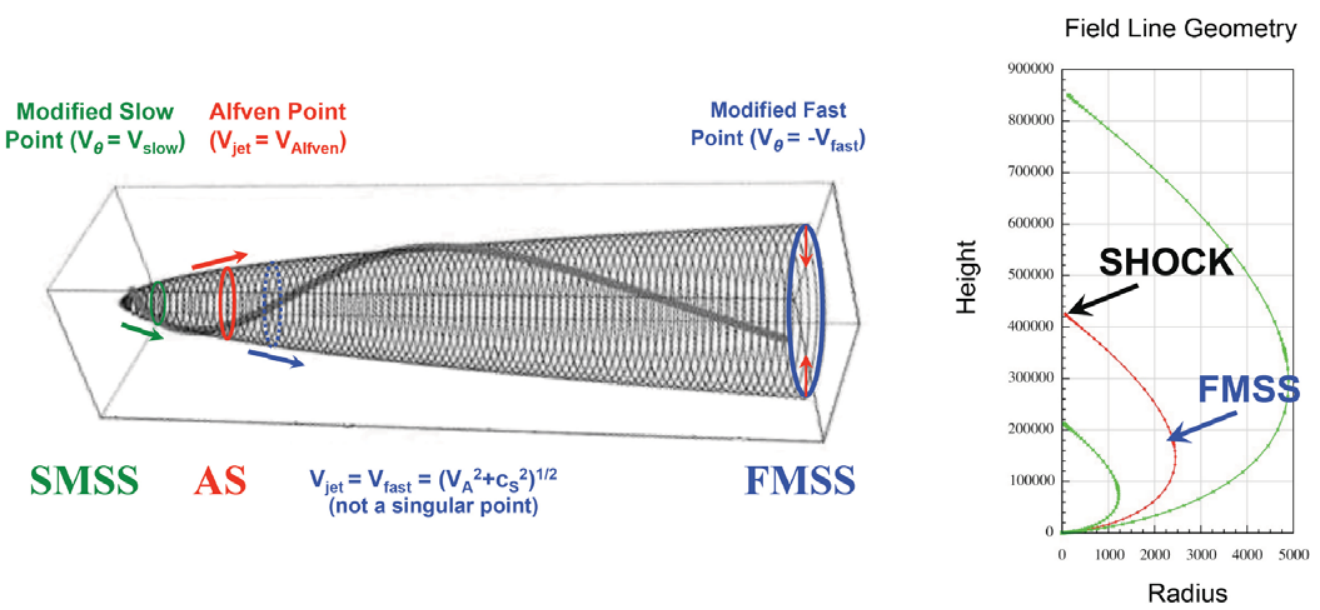

Figure 1. Two views of an accelerating jet model. Left: diagram of the jet magnetic field (tight coil), example plasma trajectory (heavy grey line), and schematic positions of the singular points/surfaces (SMSS, AS, FMSS). Right: three poloidal field/stream lines from a relativistic self-similar model that cross all three singular points, showing the position of the FMSS and possible shock. Also note that the cylindrical radial axis is stretched by a factor of 100 over the vertical axis. 
The first work to produce jet models that cross all three separatrix surfaces is Polko et al. (2010) (see also Polko's paper in this volume). The recollimation of Polko's field/ stream lines toward the axis is quite evident. Recollimation is also evident in the nonrelativistic models of Vlahakis et al. (2000) and in the relativistic simulation of McKinney (2006). These cases are all examples of a well-known device used in terrestrial jet engines to power supersonic aircraft - the convergent nozzle. The pinching effect of the convergent nozzle accelerates jet exhaust to supersonic speeds, producing one or more recollimation shocks in the outflow (which is also causally disconnected from the jet engine itself). In an F-16 supersonic fighter, for example, the nozzle is constructed with a tapered steel iris, instead of strong magnetic field, but the principle is essentially the same.

\section{The Case for a Collimation Shock Feature in Most Cosmic Jets}

Observational evidence. It is quite possible that many, if not most, cosmic jets that we observe posses a collimation feature, perhaps even a shock, shortly after their flow becomes causally disconnected from the central engine. There are, in fact, many observational clues and some strong evidence that this is indeed the case.

The first clue comes from images of FR II radio lobes and from early HD and MHD simulations of jet propagation, which show that radio lobes are consistent with the jets that feed them being weakly magnetized (Norman et al. 1982) — well below equipartition - and inconsistent with the jet structures expected from strongly magnetized flows. $\uparrow$ Yet, as we have discussed above, jets are believed to be accelerated and collimated by strong magnetic fields. If so, then the initial FR II jet created in the galactic nucleus must have lost its strong field somehow - probably closer to the black hole than we usually probe with VLBI (i.e., $<<10^{4} r_{g} \approx 1 \mathrm{pc}$ ).

In FR I type objects the collimation feature may be farther out and may already have been imaged in some sources. Cheung et al. (2007) report a speed of $<0.25 c$ for the $d$ component of HST-1 in M87, which lies $\sim 10^{6} r_{g}(120 \mathrm{pc})$ from the central black hole. Yet other components before and after this one show apparent speeds of $4-6 c$ (see Asada et al., this volume). This argues for the HST-1d region possessing a collimation shock, where the character of the relativistic jet could undergo significant changes. Similarly, BL Lac shows a strong shock at $\sim 10^{5} r_{g}$, in which a $\gamma$-ray flare was observed (Marscher et al. 2008).

Stellar mass black hole jets also show possible evidence of collimation features. $\gamma$ ray burst jets indicate possible "violent dissipation at $\sim 1000 r_{g}$ " (see Lyubarsky, this volume). And most convincingly, broad band analyses of X-ray binary jets indicate the presence of shocks that accelerate particles to relativistic energies at a distance of $100-$ $1000 r_{g}$ from the black hole (Markoff \& Nowak 2004; and Markoff, this volume).

Predicted observational features. What behavior might we expect at an MHD recollimation shock? Surprisingly, MHD collimation shocks were studied with non-relativistic simulations in the 1980s (Clarke et al. 1986; Lind et al. 1989, hereinafter LPMB). LPMB Figs. 6 \& 9 show a strong, toroidal magnetic field dominated, super-magnetosonic jet injected from the left and a strong pinch shock at $Z \approx 200$. For $Z>200$ the jet flow considerably broadens, by almost an order of magnitude, but remains forward-flowing, although slowed to trans-magnetosonic speeds in a "nose-cone" structure. In the region $Z \sim 150-200$ a magnetic chamber-like structure forms, ejecting multiple jet pulses at speeds greater than the slow magnetosonic speed, creating pairs of forward- and reverseslow shocks that propagate within the nose cone. This general structure was confirmed

$\dagger$ Note that this does not preclude the hot spots themselves [the jet Mach disks] from enhancing the magnetic field locally up to equipartition via MHD turbulence or some other process. 
for the relativistic case by Komissarov (1999). This collimation feature will have consequences for the downstream flow in the jet on kiloparsec scales and greater. If it is strong (e.g., a shock that randomizes and dissipates the strong magnetic field), a weaklymagnetized, hydrodynamic-like flow could result (as in FR IIs). However, if the feature is relatively weak (e.g., a bounce that largely preserves the strong toroidal field), then the downstream flow could continue to be current-carrying and subject to current-driven instabilities, such as helical kinks, etc. (Nakamura \& Meier 2004). Nakamura et al. (2010) argue that 1$)$ the magnetic field in this feature remains strong $\left(\beta_{\mathrm{p}} \sim 1\right.$ in the HST-1c component and $\sim 0.1$ downstream), and 2) the wiggles in the M87 jet well downstream of HST-1 are due to current-driven (and not Kelvin-Helmholtz) instabilities. The implication is that some FR I jets may remain current-carrying on the kiloparsec scale, even though FR II jets appear to be weakly-magnetized flows throughout most of their length.

\section{Summary and Conclusions}

There are some observational indications that black hole spin may play a significant role in producing jets seen in supermassive systems (active galactic nuclei [AGN]). Both observationally and theoretically, it appears that powerful, BZ-type jets may be produced by rapidly-rotating, retrograde black holes (spinning in the opposite direction to their accretion flow), while prograde ones produce radio quiet or silent sources. Not only is this "modified spin paradigm" a possible explanation for the radio loud/quiet dichotomy, it also is consistent with our current knowledge of the spin state of AGN.

In stellar-mass (X-ray binary) systems, however, it is much less clear if black hole spin plays any role. While this could indicate a fundamental difference between stellar and supermassive systems, it also could be due to gross errors in spin measurements or to observational bias against detecting the highly beamed relativistic jet expected from the BZ process.

It is clear that the rotating accretion flow does play an important role in the production of relativistic jets. And growing evidence, both observational and theoretical, indicates that jets from accretion disks are launched from a hot, geometrically thick ADAF-like state. A dramatic factor of $\sim 100$ decrease in the power of this jet (when the accretion flow goes into a cool, geometrically thin state) continues to be a viable model for the suppression of X-ray binary jets in the soft state. However, we also have identified a (factor of $\sim 20$ ) mini-suppression that can occur in the hard state when some ADAFs develop an MDAF near the black hole. This may be a viable model for hard state "outliers", if a strong correlation can be established between outliers and sources possessing bandwidthlimited noise and a strong LF QPO.

Jet acceleration and collimation is very likely achieved via rotating magnetic fields anchored either in the accretion flow or in the black hole horizon itself. While this certainly is a 3-dimensional process, self-similar jet theory can elucidate much of the physics. Specifically, different forces conspire at different stages to accomplish the acceleration: gas pressure below the slow point, magneto-centrifugal effects up to the Alfvén point, magnetic pressure up to the fast point, and pinch forces up to the modified fast point. Beyond that the flow becomes causally disconnected from the engine, and at the same time is converging toward the rotation axis at a speed greater than magnetosonic. It is, therefore, likely that the jet flow will bounce, or even shock, after it becomes a free, causally disconnected jet.

There also are numerous observational indications that many, perhaps most, cosmic jets under go a shock or other collimation feature. Depending on the strength of this feature the jet could remain current-carrying (and unstable to helical kink modes) or 
dissipate its magnetic field and become a weakly-magnetized, hydrodynamic-like flow. These processes may play a role in the morphological structure of FR I and II sources.

Acknowledgement. This research was carried out at the Jet Propulsion Laboratory, California Institute of Technology, under contract with the National Aeronautics and Space Administration.

\section{References}

Blandford, R. D. \& Payne, D. G. 1982, MNRAS, 199, 883 (BP)

Blandford, R. D. \& Znajek, R. L. 1977, MNRAS, 179, 433 (BZ)

Bogovalov S. V. 1994, MNRAS, 270, 721

Brenneman, R. D. \& Reynolds, R. L. 2006, ApJ, 652, 1028

Cheung, C. C. et al. 2007, ApJ, 663, L65

Clarke, D. A. et al. 1986, ApJ, 311, L63

Elvis, M. et al. 2002, ApJ, 565, L75

Fabian, A. C. et al. 2009, Nature, 459, 540

Fender, R. P. 2001, in Black Holes in Binaries and Galactic Nuclei (Heidelberg: Springer-Verlag), ed. L. Kaper, E. P. J. van den Huevel, \& P. A. Woudt, p. 193

Fender, R. P. et al. 2004, MNRAS, 355, 1105

Fender, R. P. et al. 2010, MNRAS, 4061425

Fragile, P. C. \& Meier, D. L. 2009, ApJ, 693, 771

Garofalo, D. 2009, ApJ, 699, 400

Garofalo, D. et al. 2010, MNRAS, 406, 975

Hawley, J. F. \& Krolik, J. H. 2006, ApJ, 61, 103

Iwasawa, D. et al. 1996, MNRAS, 282, 1038

Kataoka, J. et al. 2007, PASJ, 59, 279

Komissarov, S. S. 1999, MNRAS, 308, 1069

Li, Z.-Y. et al. 1992, ApJ, 394, 459

Lind, K. R. et al. 1989, ApJ, 344, 89

Lynden-Bell, D. 1996, MNRAS, 279, 389

Markoff, J. C. \& Nowak, C. F. 2004, ApJ, 609, 976

McClintock, J. E. \& Remillard, R. A. 2006, in Compact stellar X-ray sources (Cambridge: Cambridge Univ. Press), ed. W. Lewin \& M. van der Klis, p. 157

McKinney, J. C. 2006, MNRAS, 368, 1561

McKinney, J. C. \& Gammie, C. F. 2004, ApJ, 611, 977

Meier, D. L. et al. 1997, Nature, 388, 350

Meier, D. L. 1999, ApJ, 522, 753

Meier, D. L. 2005, Ap $\& S S, 300,55$

Meier, D. L. \& Garofalo, D. 2010, in preparation.

Michel, F. C. 1969, ApJ, 158, 727

Migliari, S. \& Fender, R. P. 2006, MNRAS, 366, 79

Nakamura, M. \& Meier, D. L. 2004, ApJ, 617, 123

Nakamura, M. et al. 2010, ApJ, 721, 1783

Polko, P. et al. 2010, ApJ, 723, 1343

Pringle, J. E. 1976, MNRAS, 177, 65

Punsly, B. \& Coroniti, F. V. 1990, ApJ, 354, 583

Romanova, M. M. et al. 1998, ApJ, 500, 703

Sambruna, R. M. et al. 2009, ApJ, 700, 1473

Shakura, N. I. \& Sunyaev, R. A. 1976, MNRAS, 175, 613

Sikora, M. et al. 2007, ApJ, 658, 815

Szuszkiewicz, E. \& Miller, J. C. 2001, MNRAS, 328, 36

Uzdensky, D. A. 2005, ApJ, 620, 889

Uzdensky, D. A. \& MacFadyen, A. I. 2006, ApJ, 647, 1192

Vlahakis, N. et al. 2000, MNRAS, 318, 417 
Vlahakis, N. \& Konigl, A. 2003, ApJ, 596, 1080

Wilson, A. S. \& Colbet, E. J. M. 1995, ApJ, 438, 62

Yu, Y. \& Tremaine, S. 2002, MNRAS, 335, 965

\section{Discussion}

PE'ER: You spoke about Blandford-Znajek mechanism for jet formation. However, we know that jets exist in neutron stars, and possibly white dwarfs systems. So, what is the jet launching mechanism in these systems?

MEIER: First, as Dr. Gallo reported, there is yet no evidence that BH spin is important in microquasars. So, for microquasars it is possible that the jets are disk driven (BlandfordPayne process). I would argue, however, that there is some evidence thath the BZ process is important in radio loud AGNs. In the case of mocroquasars with neutron stars, a diskdriven jet (BP process) is a reasonable model. However, these systems also are quenched at $\dot{M} \sim 0.01$, but not by a factor of 30 or more. Instead, the jet partially recovers and continues to increase in strength with $\dot{M}$, albeit at a level perhaps 10 times lower than for $\dot{M}<0.01$. For this range $(\dot{M}<0.01)$, I would suggest that this weaker jet is powered by the rotating neutron star and the "propeller" mechanism.

GHISELLini: You supposed that jets were likely to be formed at low $\dot{m}$, but recent results on blazars suggest that they exist at all $\dot{m}$, and that the jet power is proportional to the accretion rate

MEIER: My suggestion was that jets are produced when the accretion flow is in a hard, geometrically thick state. This does not mean that the source is not accreting at a high state, only that for a short period o time the source enters a temporarily hot accretion phase to eject a jet component, but then returns to a softer disk state (which temporarily turns off the jet again). This all would be possible if the accretion flow were unsteady and the disk wew undergoing disk instabilities - just like those instabilities expected as the accretion rate approaches Eddington.

SIKORA: What can be the mechanism/scenario of loading of Blandford-Znajek jets by protons, presence of which in extragalactic jets is indicated by several independent observations?

MEIER: Mass loading, of course, is a big problem in the theory of jets of all kinds. Some possibilities are loading from the accretion flow into the launched jet, loading by collision of the jet with the broad line clouds or other material in the ISM, a entrainment of material at a collimation shock, or entrainment at the interface between the jet and the ambient medium. There also may be a wind surounding the jet that could carry baryons which would be entrained downstream. I am not currently familiar with the observational constraints to judge which of these are plausible and which are not. 